\title{
BLOG DA PROMOÇÃO DA SAÚDE RELATO DE EXPERIÊNCIA SOBRE A PRODUÇÃO DE UM OBJETO DE APRENDIZAGEM
}

\author{
Camilo Darsie de Souza - UFRGS - camilods@terra.com.br \\ Élen Jiovana Oliveira da Rocha - UFRGS - elenjorocha@yahoo.com.br \\ Marisol Silveira de Oliveira - HCPA - marisol.sol@gmail.com \\ Mára Lúcia Fernandes Carneiro - UFRGS - mara.napead@ sead.ufrgs.br \\ Roberta Alvarenga Reis - UFRGS - robertaalvarengareis.ead@gmail.com \\ Cristianne Maria Famer Rocha - UFRGS - cristianne.rocha@terra.com.br
}

\begin{abstract}
Resumo
Este artigo é constituído por um relato de experiência ligado à elaboração de um objeto de aprendizagem, no ano de 2011. Tal objeto de aprendizagem abrigou documentos e recursos ligados à Promoção da Saúde com o objetivo de se estabelecer um espaço de discussão capaz de promover a compreensão do conceito mencionado e a interação entre os usuários da ferramenta. Como forma de validar o objeto de aprendizagem, ao final do ano, foi aplicado um questionário de avaliação que contou com a participação de cerca de 100 usuários. Isso oportunizou aos organizadores uma visão ampla acerca da importância deste tipo de ferramenta no contexto da Educação em Saúde. Assim, o objetivo do texto é apresentar os caminhos percorridos pelo grupo de organizadores e ressaltar a importância do uso de tecnologias para a Educação em Saúde.
\end{abstract}

Palavras-chave: objeto de aprendizagem, promoção da saúde, educação.

\section{BLOG OF HEALTH PROMOTION EXPERIENCE REPORT ON THE PRODUCTION OF AN OBJECT OF LEARNING}

\begin{abstract}
This paper consists of an experience report on the development of a learning object, in the year of 2011. This object of learning hosted documents and resources related to Health Promotion with the aim of establishing a forum for discussion that promotes understanding of the concept mentioned and interaction among the users of the tool. In order to validate the learning object a questionnaire was administered assessing about 100 users at the end of the year. This research gave the organizers a broader view about the importance of such a tool in the context of Health Education. The aim of the paper is to present the paths taken by the group of organizers and the importance of using such technologies for Health Education.
\end{abstract}

Keywords: object of learning, health promotion, education. 


\section{Introdução}

Nos dias de hoje podem ser observadas grandes transformações no que diz respeito ao Sistema Único de Saúde (SUS), as quais motivam - ao mesmo tempo em que são garantidas por tais - as iniciativas que envolvem a Educação em Saúde. Com isso, projeta-se grande responsabilidade sobre os centros de formação técnica e sobre as instituições acadêmicas que se propõem a formar e a atualizar profissionais que trabalham na área da saúde. Emerge, então, a necessidade de serem pensadas questões relacionadas às formas e aos objetivos do ensino, à estrutura curricular, às práticas pedagógicas e aos conteúdos apresentados (CAVALCANTE; VASCONCELLOS, 2007).

Considerando essa situação, bem como sua relevância e associando-a ao fato de que as transformações relacionadas à Educação vêm ocorrendo de forma rápida e intensa, é relevante se levar em conta que as práticas que envolvem a Educação em Saúde transformam e são transformadas pelas ações de outras áreas. Ainda, é pertinente destacar que muitas dessas mudanças, e das possibilidades que podem ser vislumbradas no que se refere ao aprimoramento de novas formas de ensino e de aprendizagem na área da saúde se relacionam, entre outras coisas, ao igualmente rápido e intenso desenvolvimento da internet nas últimas duas décadas.

A partir disso, salienta-se que desde o início da década de 1990, o uso da internet tem se tornado cada vez mais comum e, com isso, novas maneiras de acesso a informações, que antes poderiam ser limitadas, devido às suas formas de circulação, têm se propagado de modo a alcançar diferentes indivíduos que as buscam por necessidades profissionais ou, simplesmente, por lazer. As tecnologias da informação e da comunicação perpassam e se articulam às mudanças sociais que ocorrem contemporaneamente. Dessa maneira, a utilização das informações disponíveis na rede mundial de computadores faz com que as sociedades se apropriem dos conhecimentos mais diversos e, portanto, a internet representa uma importante ferramenta ligada à aprendizagem. (SARAIVA, 2010).

Appadurai (2005) argumenta que os meios de comunicação eletrônicos, entre eles a internet, aproximam as pessoas de informações que, em outros tempos, não seriam alcançáveis. Neste contexto, os textos têm tomado novas formas devido às inovações existentes para a divulgação das informações que fazem com que, a partir de interesses individuais, diferentes caminhos sejam seguidos na produção de um texto complexo que é formulado pelas muitas informações disponíveis na internet. (BAUMAN, 2001). Para Lévy (1996), a internet oportuniza a fabricação de hipertextos, os quais são configurados pela não linearidade de leitura que a internet proporciona através de seu seus atalhos. Nesta direção, é possível ser dito que os ambientes virtuais podem servir como importantes facilitadores no que diz respeito ao aumento da potencialidade de aprendizagens de forma autônoma.

$\mathrm{Na}$ esteira dessas transformações, tomam forma os chamados 'objetos de aprendizagem virtuais' que podem ser definidos como ferramentas digitais cuja atualização constante, bem como a combinação com outras ferramentas marcam o dinamismo de tais recursos. Os objetos de aprendizagem podem ser definidos como sendo recursos digitais que são utilizados e criados para servirem como apoio no que diz respeito aos processos de aprendizagem. Assim, qualquer ferramenta digital, formada por pequenos conjuntos de recursos, que seja estabelecida para ser usada pedagogicamente se enquadra na definição de objeto de aprendizagem (WILEY, 2000). Tarouco et.al. (2004) explicam que os objetos de aprendizagem proporcionam formação 
continuada, cuja qualidade pode ser garantida, entre outros aspectos, pela potencialização de situações de aprendizagem significativas, visto que conta com o interesse daquelas pessoas que acessam tais ferramentas.

É relevante chamar a atenção para o fato de que muitos pesquisadores defendem a ideia de que os blogs não se configuram como OA devido ao fato de serem, em princípio, diários virtuais. Da mesma forma que um hipertexto ou uma animação, em si, não se constituem como objetos de aprendizagem. No entanto, um blog, hipertexto ou animação, quando estruturados de forma a proporcionar conteúdo significativo, atividades e ou espaços de interação, podem apoiar a aprendizagem e serem considerados com objetos de aprendizagem.

Neste sentido, o Blog da Promoção da Saúde foi desenvolvido para servir como fonte de materiais de referência - textos, vídeos, entrevistas, documentos - que possibilitam aos seus usuários estudar questões relacionadas à Promoção da Saúde ao mesmo tempo em que garante o acesso a outras fontes de informação. Além disso, o formato 'blog' permite que os usuários registrem comentários e tenham acesso a diversos recursos que oportunizam a interatividade entre os organizadores e os usuários, potencializando a comunicação e a troca de experiências e recursos entre tais atores, o que facilita a compreensão do conceito de Promoção da Saúde.

Com isso, o objetivo deste artigo é relatar e analisar a experiência dos autores/organizadores no processo de construção de um objeto de aprendizagem que visa oportunizar situações de aprendizagem significativas aos seus usuários, tendo como princípio as discussões mais atuais relacionadas à Educação em Saúde e a necessidade de serem pensados e criados novos e criativos recursos para a formação de profissionais que atuam ou atuarão na área.

\section{Contexto e objetivos que deram origem ao objeto de aprendizagem}

O 'Blog da Promoção da Saúde: Coletânea de recursos e experiências' é um objeto de aprendizagem que foi criado no âmbito do Edital $\mathrm{n}^{\circ} 15$ da Secretaria de Educação a Distância da Universidade Federal do Rio Grande do Sul (SEAD/UFRGS) e visa disponibilizar para docentes, discentes e comunidade em geral, informações úteis sobre o tema da Promoção da Saúde. O objetivo principal do Blog é criar oportunidades de acesso a diversos materiais relacionados à Promoção da Saúde e, ao mesmo tempo, estabelecer um foro de discussão sobre o tema, no qual diferentes atores podem participar para ampliar seus conhecimentos e práticas em relação aos temas apresentados, desde de diferentes perspectivas. Para dar conta da empreitada, de modo a produzir um objeto de aprendizagem que pudesse atingir os objetivos desejados, de forma eficaz, foi formado um grupo de trabalho com docentes e discentes oriundos de diferentes áreas do conhecimento (Saúde, Educação, Informática e Comunicação).

Assim, o Blog da Promoção da Saúde foi desenvolvido de modo a se configurar como um objeto de aprendizagem interativo, didático, atualizado, original e que possa servir como referência aos docentes, discentes e usuários em geral, de tal forma que todos possam fazer uso das informações existentes para analisar e discutir ações, atividades, recursos e ferramentas que propiciem maior uso da Promoção da Saúde no cotidiano, enquanto profissionais (ou futuros profissionais) da área, mas também enquanto usuários do Sistema Único de Saúde (SUS).

\section{Como o Blog da Promoção da Saúde tomou forma}


Tendo sido apresentadas algumas informações gerais sobre o Blog da Promoção da Saúde, passa-se agora para a descrição das etapas que fizeram parte do processo de construção do objeto de aprendizagem.

Em um primeiro momento, após aprovação do projeto, foram desenvolvidas atividades que envolveram a procura, a análise e a seleção de outros objetos de aprendizagem similares, que, de certo modo, balizaram a construção do OA apresentado aqui. Essas investigações foram feitas com maior atenção durante o início do trabalho, no entanto, não foram deixadas de lado até o presente momento devido à necessidade de atualização constante em relação às novas possibilidades que podem ser encontradas em ferramentas semelhantes. Além disso, textos, revistas, vídeos, imagens e outros tipos de materiais foram selecionados - e continuam sendo - para a composição do conteúdo disponibilizado para consulta por parte dos usuários.

A produção e a edição do Blog foram feitas quase que em concomitância com a seleção de materiais e, portanto, desde cedo o OA se encontrou disponível na rede mundial de computadores para consulta e, na medida do possível, para ser avaliado por seus usuários que colaboraram a partir de mensagens enviadas através do próprio ambiente virtual. Para tanto, foi criada uma identidade visual (logomarca, cores, formatação) que envolve os conhecimentos construídos no âmbito dos cursos da área da saúde da Universidade Federal do Rio Grande do Sul. Além disso, foram feitas reuniões regulares, nas quais se discutiu as sugestões de docentes e discentes de cursos da área da Saúde. Nessas reuniões, novas propostas de formatação do Blog foram sendo estudadas e desenvolvidas, além de terem sido feitas escolhas no que se refere a novos documentos e demais recursos que foram sendo incorporados ao conjunto de materiais disponibilizado.

É relevante ser mencionado que durante todo o processo de produção do conjunto de informações que compõem o Blog, foi bastante grande a interatividade entre os organizadores e, ainda, entre estes e os diversos usuários que, ao usufruir da ferramenta virtual, fizeram colocações e solicitações pertinentes que oportunizaram resultados mais interessantes, do ponto de vista dos usuários. Dentre esses resultados positivos podem ser mencionados, em primeiro lugar, o próprio conteúdo que constitui o objeto de aprendizagem, pois conforme as sugestões de usuários bem como a solicitação de docentes que fizeram do objeto de aprendizagem parte de suas aulas foi possível ser criado um histórico de materiais atualizados e, efetivamente, relevantes. Em segundo lugar, os próprios organizadores puderam compartilhar conhecimentos distintos e, com isso, questões sobre o tema da Promoção da Saúde puderam ser melhor entendidas por todos.

No entanto, uma das dúvidas que acompanhou todo o processo de construção deste ambiente virtual diz respeito à relevância dele no que se refere, principalmente, à comunidade acadêmica envolvida nas discussões que dão forma à Educação em Saúde e às possíveis transformações que isso pode acarretar em relação ao cotidiano. Portanto, como forma de validar o objeto de aprendizagem, bem como, de registrar um número significativo de opiniões e contribuições acerca do Blog da Promoção da Saúde, foi realizado um processo de validação, a seguir explicitado.

\section{Sobre o processo de validação}

Foi elaborado um questionário com 12 (doze) perguntas objetivas a partir das quais os participantes da pesquisa avaliaram diferentes aspectos ligados à ferramenta. Entre os aspectos avaliados pelos internautas que responderam ao questionário podem ser apontados a relevância desse objeto de aprendizagem para as necessidades discentes, 
docentes e do público em geral, a identidade visual do Blog e a qualidade e relevância dos diferentes materiais disponibilizados. Além disso, duas questões abertas foram elaboradas de modo a oportunizar manifestações ligadas a sugestões que envolvessem o aperfeiçoamento da ferramenta produzida. Tais questões, foram disponibilizadas no próprio Blog, durante o mês de novembro, e os convites foram lançados via e-mail para diversas listas de contatos que abrangeram diferentes modalidades de usuários - em especial, docentes e discentes de cursos da área da Saúde e da Educação, no Brasil e no exterior. Assim, ao final do processo de validação do Blog foram obtidas as opiniões de 100 (cem) usuários ${ }^{3}$, cujos resultados são apresentados na Tabela 1.

Tabela 1 - Distribuição das respostas dos usuários do Blog Promoção da Saúde quanto a relevância deste Objeto de Aprendizagem. 2011

\begin{tabular}{|l|c|c|c|c|c|}
\hline & $\begin{array}{c}\text { Muito } \\
\text { Relevante } \\
\text { Ou Muito } \\
\text { Bom }\end{array}$ & $\begin{array}{c}\text { Relevante } \\
\text { ou } \\
\text { Bom }\end{array}$ & $\begin{array}{c}\text { Pouco } \\
\text { Relevante } \\
\text { ou } \\
\text { Regular }\end{array}$ & $\begin{array}{c}\text { Nada } \\
\text { Relevante } \\
\text { ou } \\
\text { Ruim }\end{array}$ & $\begin{array}{c}\text { Indiferente } \\
\text { ou } \\
\text { Não sei }\end{array}$ \\
\hline $\begin{array}{l}\text { Você considera relevante a utilização de } \\
\text { objetos de aprendizagem (tal como o } \\
\text { Blog da Promoção da Saúde) como } \\
\text { recurso pedagógico? }\end{array}$ & $77 \%$ & $22 \%$ & $0 \%$ & $0 \%$ & $1 \%$ \\
\hline $\begin{array}{l}\text { No que diz respeito às suas atividades } \\
\text { (docentes, discentes, profissionais, } \\
\text { outras), qual o nível de relevância do } \\
\text { Blog da Promoção da Saúde? }\end{array}$ & $63 \%$ & $33 \%$ & $2 \%$ & $0 \%$ & $2 \%$ \\
\hline
\end{tabular}

No que diz respeito à importância de ferramentas digitais desta ordem, é possível se observar, pelas respostas dadas pelos usuários, que tais recursos são significativos no processo de aprendizagem. Considerando esta realidade, os organizadores puderam vislumbrar possibilidades ligadas à manutenção do OA, de modo a continuar a postagem de informações que possam ser úteis aos docentes e discentes das áreas que são atravessadas pelas questões que envolvem a Promoção da Saúde. Além disso, a divulgação do OA, junto aos docentes e aos grupos de discentes foi motivada. É possível afirmar que o Blog da Promoção da Saúde tem sido utilizado por novos usuários que tiveram seus primeiros contatos com a ferramenta no primeiro semestre de 2012, a partir de indicações de professores e colegas que já a conheciam.

Um exemplo dessa situação, se configura a partir da disciplina de Educação e Saúde, da Universidade Federal do Rio Grande do Sul, direcionada aos estudantes de cursos da área da saúde - Enfermagem, Fisioterapia, Educação Física, etc. Muitos desses estudantes encontram-se no primeiro semestre de suas formações e, a partir da indicação do docente da disciplina, formalmente, encontram no OA um espaço onde textos e documentos indicados para as aulas se encontram disponíveis. O Blog também é indicado em disciplinas teóricas $\left(1^{\circ}\right.$ semestre $)$ e ambientes virtuais de estágio em Saúde Coletiva $\left(5^{\circ}, 7^{\circ}\right.$ e $8^{\circ}$ semestres) do Curso de Fonoaudiologia.

Tabela 2 - Distribuição das respostas dos usuários do Blog Promoção da Saúde quanto a estrutura e apresentação do OA. 2011

\begin{tabular}{|l|c|c|c|c|c|}
\hline & $\begin{array}{c}\text { Muito } \\
\text { Relevante } \\
\text { Ou } \\
\text { Muito Bom }\end{array}$ & $\begin{array}{c}\text { Relevante } \\
\text { ou } \\
\text { Bom }\end{array}$ & $\begin{array}{c}\text { Pouco } \\
\text { Relevante } \\
\text { ou } \\
\text { Regular }\end{array}$ & $\begin{array}{c}\text { Nada } \\
\text { Relevante } \\
\text { ou } \\
\text { Ruim }\end{array}$ & $\begin{array}{c}\text { Indiferente } \\
\text { ou } \\
\text { Não sei }\end{array}$ \\
\hline $\begin{array}{c}\text { O que você achou da apresentação (design, } \\
\text { logomarca, distribuição dos materiais, etc.) }\end{array}$ & $57 \%$ & $41 \%$ & $1 \%$ & $0 \%$ & $5 \%$ \\
\hline
\end{tabular}




\begin{tabular}{|l|l|l|l|l|l|}
\hline do Blog da Promoção da Saúde? & & & & \\
\hline $\begin{array}{l}\text { O que você achou da distribuição das } \\
\text { informações dentro da página do Blog? }\end{array}$ & $43 \%$ & $49 \%$ & $5 \%$ & $0 \%$ & $3 \%$ \\
\hline
\end{tabular}

Mesmo tendo sido consideradas positivas, as respostas relacionadas à estrutura do OA (Tabela 2) oportunizaram aos organizadores momentos de reflexão a respeito da forma como os materiais foram apresentados dentro do espaço virtual. Deste modo, as modalidades de recursos foram revisadas e novas formas de distribuição foram criadas. Para tanto, novas guias de conteúdos foram disponibilizadas de maneira a deixar mais claras as informações bem como os materiais constantes no Blog. Certos recursos, de acordo com os interesses manifestados na avaliação (Tabela 3) receberam destaque, pois os organizadores consideraram ser fundamental facilitar o acesso aos conteúdos apontados pelos usuários como mais relevantes.

Entre os recursos mais relevantes, podem ser destacados, os links de acesso aos diversos sites nacionais e internacionais que abordam o tema. É importante considerar que a postagem de conteúdos foi feita tendo como princípio o respeito pelas fontes dos materiais, assim, o fato de haver conexão entre o Blog da Promoção da Saúde e outros ambientes virtuais semelhantes, possibilitou um aumento significativo no que diz respeito ao aumento de materiais disponíveis. Também foram consideradas importantes de acordo com os avaliadores do OA outras modalidades de materiais como: artigos científicos, vídeos, dissertações e teses. Tais modalidades receberam especial atenção, principalmente porque a colaboração dos avaliadores permitiu a inclusão de novos materiais.

Tabela 3 - Distribuição das respostas dos usuários do Blog Promoção da Saúde quanto ao material disponibilizado no OA

\begin{tabular}{|l|c|c|c|c|c|}
\hline & $\begin{array}{c}\text { Muito } \\
\text { Relevante } \\
\text { Ou } \\
\text { Muito Bom }\end{array}$ & $\begin{array}{c}\text { Relevante } \\
\text { ou } \\
\text { Bom }\end{array}$ & $\begin{array}{c}\text { Pouco } \\
\text { Relevante } \\
\text { ou } \\
\text { Regular }\end{array}$ & $\begin{array}{c}\text { Nada } \\
\text { Relevante } \\
\text { ou } \\
\text { Ruim }\end{array}$ & $\begin{array}{c}\text { Indiferente } \\
\text { ou } \\
\text { Não sei }\end{array}$ \\
\hline $\begin{array}{l}\text { O que você achou dos tipos de materiais } \\
\text { (textos, vídeos, imagens) disponíveis no } \\
\text { Blog? }\end{array}$ & $54 \%$ & $40 \%$ & $1 \%$ & $0 \%$ & $5 \%$ \\
\hline $\begin{array}{l}\text { O que você achou dos sites cujo acesso é } \\
\text { facilitado pelos links disponíveis no Blog? }\end{array}$ & $70 \%$ & $29 \%$ & $0 \%$ & $1 \%$ & $0 \%$ \\
\hline $\begin{array}{l}\text { O que você achou dos vídeos disponíveis } \\
\text { no Blog? }\end{array}$ & $49 \%$ & $47 \%$ & $0 \%$ & $0 \%$ & $4 \%$ \\
\hline $\begin{array}{l}\text { O que você achou das imagens associadas } \\
\text { à Promoção da Saúde disponíveis no Blog? }\end{array}$ & $45 \%$ & $44 \%$ & $5 \%$ & $2 \%$ & $4 \%$ \\
\hline $\begin{array}{l}\text { O que você achou dos artigos disponíveis } \\
\text { no Blog? }\end{array}$ & $56 \%$ & $41 \%$ & $1 \%$ & $0 \%$ & $2 \%$ \\
\hline $\begin{array}{l}\text { O que você achou das dissertações e teses } \\
\text { disponíveis no Blog? }\end{array}$ & $51 \%$ & $42 \%$ & $2 \%$ & $0 \%$ & $5 \%$ \\
\hline $\begin{array}{l}\text { O que você achou das revistas disponíveis } \\
\text { no Blog? }\end{array}$ & $55 \%$ & $38 \%$ & $0 \%$ & $0 \%$ & $7 \%$ \\
\hline $\begin{array}{l}\text { O que você achou das demais publicações } \\
\text { disponíveis no Blog? }\end{array}$ & $47 \%$ & $47 \%$ & $3 \%$ & $0 \%$ & $3 \%$ \\
\hline
\end{tabular}

As questões abertas trouxeram sugestões importantes, como, por exemplo, a necessidade de diversificação de autores no que diz respeito aos artigos e textos 
disponibilizados, a importância de diferenciação entre discussões que envolvem a Promoção da Saúde e a Prevenção de Doenças e, principalmente, a necessidade de se ampliar as possibilidades de divulgação e de interconexão a outros objetos de aprendizagem semelhantes ao Blog no que diz respeito ao seu uso por um número ainda maior de pesquisadores. Além disso, questões relacionadas à necessidade de disponibilização de espaços de interação, como bate-papo ou fórum, para propiciar a discussão sobre tópicos específicos. Outro ponto que chamou a atenção dos organizadores foi a demanda por divulgação de eventos da área da saúde, fato que pode significar que o Blog auxilia não apenas na busca por materiais específicos, mas representa um importante espaço de comunicação sobre atividades ligadas à área da Saúde.

Tendo em vista essas questões, as alterações necessárias foram avaliadas e, de acordo com as possibilidades técnicas, agregadas ao objeto. Algumas das sugestões estão em fase de análise, para que sejam contempladas nos próximos meses, mas mesmo assim, na medida do possível, a maior parte dos pedidos feitos pelos avaliadores foi atendida.

\section{Considerações Finais}

No geral, de acordo com o que pode ser observado, a partir dos dados obtidos, é possível se considerar que o Blog da Promoção da Saúde foi avaliado positivamente pelos participantes da pesquisa. A maioria das respostas aponta para a relevância do objeto de aprendizagem, da mesma forma que sinaliza a qualidade dos materiais disponibilizados. Isso significa que tal modalidade de ferramenta pedagógica se faz válida para as atividades que envolvem a Educação em Saúde. Além disso, é interessante se pensar que o objetivo que norteou o processo de validação proposto limitado à avaliação da ferramenta sob o enfoque do seu uso e objetivos - pode dar origem a novos questionamentos e a novas análises ligadas, por exemplo, à qualidade dos materiais disponibilizados e à importância da utilização desses materiais em sala de aula, bem como em relação aos critérios de usabilidade da própria interface.

É oportuno mencionar, ainda, que as vivências apresentadas neste texto, podem servir como exemplos relacionados à importância do uso de novas ferramentas no processo de ensino-aprendizagem em saúde. No caso específico da experiência apresentada aqui, pode ser dito que os OA desfrutam da capacidade de ampliar discussões para além das salas de aula e dos ambientes profissionais, de modo a oportunizar interações que auxiliam no entendimento de temas relevantes, neste caso, aqueles relacionados à Promoção da Saúde.

Para finalizar, é importante ser ressaltado que a manutenção e a atualização do Blog da Promoção da Saúde deverão ser permanentemente feitas pelos docentes e discentes envolvidos no processo de construção do OA. Acredita-se que dessa forma, tal ferramenta, possa transformar-se em uma referência no que diz respeito aos textos, documentos, vídeos, imagens entre outros, que frequentemente se mostram necessários para o desenvolvimento da noção de Promoção da Saúde.

\section{Notas}


${ }^{1}$ Um blog pode ser definido como um sistema de publicação na web destinado a divulgar informação por ordem cronológica, de forma semelhante a um diário. (SANTAROSA et. al, 2010, p. 150).

${ }^{2}$ Disponível em http://blogdapromocao-saudecoletiva.blogspot.com.br

${ }^{3}$ Apesar de a divulgação do processo de validação do Blog ter sido direcionada, principalmente, a sujeitos que frequentam ambientes universitários, não foi propósito da avaliação realizada identificar, entre os avaliadores, quem utiliza (ou utilizou) o Blog para desenvolvimento de atividades acadêmicas.

\section{Referências Bibliográficas}

APPADURAI, A. Dimensões culturais da globalização: A modernidade sem peias. Trad. Telma Costa. Lisboa: Teorema Editorial, 2005.

BAUMAN, Z. Modernidade Líquida. Rio de Janeiro: Jorge Zahar Editora, 2001.

CAVALCANTE, M.T.L.; VASCONCELLOS, Ml M. Tecnologia de informação para a educação na saúde: duas revisões e uma proposta. Ciênc. saúde coletiva [online]. 2007, vol.12, n.3, pp. 611-622. ISSN 1413-8123.

LEVY, P. O que é o Virtual?. São Paulo: ed 34, 1996.

SARAIVA, K. Educação a distância - outros tempos outros espaços. Ponta Grossa: UEPG, 2010.

SANTAROSA, L.; CONFORTO, D.; PASSERINO, L.M.; ESTABEL, L.; CARNEIRO, M.L.F.; GELLER, M. Tecnologias Digitais Acessíveis. Porto Alegre: JSM Comunicação Ltda, 2010.

TAROUCO, L.M.R.; FABRE, M.C.J. M.; GRANDO, A.R.S.; KONRATH, M.L.P. Objetos de Aprendizagem para M-Learning. Florianópolis: SUCESU - Congresso Nacional de Tecnologia da Informação e Comunicação, 2004.

WILEY, D. A. Connecting learning objects to instructional design theory: A definition, a metaphor, and a taxonomy. In D.A.Wiley (Ed.), The Instructional Use of Learning Objects, 2000. 